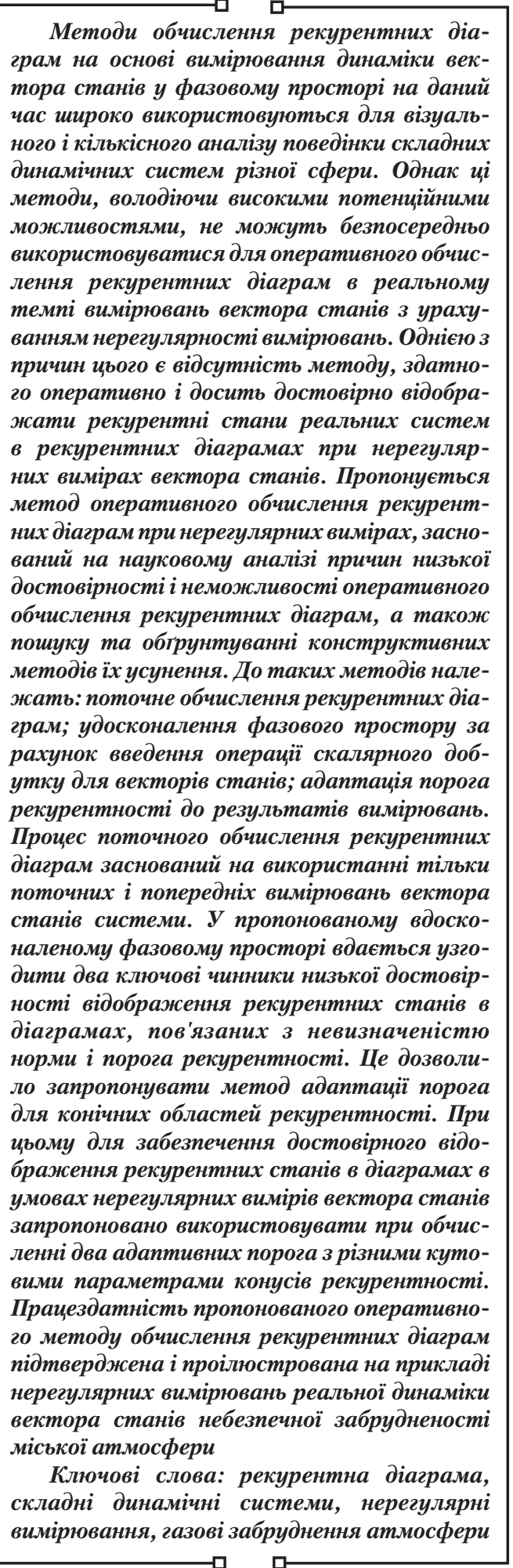

Методи обчислення рекурентних діаграм на основі вимірювання динаміки векного $і$ кількісного аналізу поведінки складних динамічних систем різної сфери. Однак иі використовуватися для оперативного обчислення рекурентних діаграм в реальному причин иъого є відсутність методу, здатно$2 о$ оперативно $i$ досить достовірно відобра жати рекурентні стани реальних систем метод оперативного обчислення рекурентних діаграм при нерегулярних вимірах, заснообчислення рекурентних діаграм, а також пошуку та обгрунтуванні конструктивних грам; удосконалення фазового простору за рахунок введення операчії скалярного добол векторів станів; адаптачія порога діаграм заснований на використанні тільки

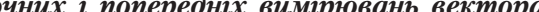
наленому фазовому просторі вдається узгодіаграмах, пов'язаних з невизначеністю норми і порога рекурентності. Це дозволидля конічних областей рекурентності. При ивому для забезпечення достовірного відображення рекурентних станів в діаграмах в ленні два адаптивних порога з різиими кутовими параметрами конусів рекурентності.

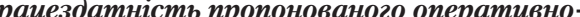
нерегулярних вимірювань реальної динаміки вектора станів небезпечної забрудненості

складні динамічні системи, нерегулярні $\square$ 口-

\section{CALCULATION THE RECURRENT DIAGRAMS FOR NON-REGULAR MEASUREMENTS \\ DEVELOPMENT OF THE METHOD OPERATIVE}

UDC 004.932

DOI: $10.15587 / 1729-4061.2019 .181516$
B. Pospelov

Doctor of Technical Sciences, Professor Department of Organization and

Coordination of Research Activities

Scientific-Methodical Center of Educational Institutions in

the Sphere of Civil Defence

Chernyshevska str., 94, Kharkiv, Ukraine, 61023

E-mail: pospelov@nuczu.edu.ua

O. Kra in i u k ov

Doctor of Geographical Sciences, Associate Professor Department of Ecological Safety and Environmental Education

V. N. Karazin Kharkiv National University

Svobody sq., 4, Kharkiv, Ukraine, 61022

A. S a v c he n k o

$\mathrm{PhD}$, Senior Research

Department of Prevention Activities and Monitoring*

S. H a r buz

$\mathrm{PhD}$

Department of Fire and Technological Safety of

Facilities and Technologies*

O. Cherkash y n

$\mathrm{PhD}$

Department of Fire and Rescue Training*

S. S h cher bak

Department of Fire and Rescue Training*

I. Rolin

Doctor of Military Sciences, Associate Professor**

V. Te m n i k v

$\mathrm{PhD**}$

*National University of Civil Defence of Ukraine Chernyshevska str., 94, Kharkiv, Ukraine, 61023

**Military Institute for Tank Troops

National Technical University "Kharkiv Polytechnic Institute"

Poltavskyi Shliakh str., 192, Kharkiv, Ukraina, 61000

\section{Introduction}

The study of most complex systems, both natural and artificial, shows that their bases are non-linear processes, and their behavior satisfies a fundamental principle of dissipative dynamical systems, that is state recurrence. The recurrence of states means that certain states of systems are repeated in time [1]. There are methods of recurrence 
plots (RP) proposed in [2] to visualize recurrence states of complex dynamic systems, RP methods are fundamental for quantitative state recurrence analysis (RQA).

Recently, researchers have widely used RP and RQA methods in various sciences and applications [1,3]. However, capabilities of RQA methods depend substantially on reliability of mapping of the recurrent behavior of real systems in RP. It is known that the reliability of mapping of recurrence states in RP depends substantially on conditions and nature of obtaining of measurement information. One of the limitations of the known methods for calculation of RP [4] is their inherent threshold uncertainty. The threshold uncertainty of the methods limits reliability of mapping of recurrence states significantly under unknown and changing conditions, which are characteristic to most applications. In this regard, the problem of improvement of methods for calculation of RP arises.

\section{Literature review and problem statement}

A base of RP calculation methods is real state measurements of complex dynamic systems. Therefore, RP methods are attractive tools for studying of a state of complex dynamic systems in various fields [2]. If obtaining of measurement information is discrete in time, a change in a vector of states of the system in the corresponding phase space will be mapped as a point, which moves discretely along a certain trajectory of system states. In this case, the recurrence of states at discrete instants will mean that arbitrary points of the trajectory turn out to be close in some sense [4]. Unlike the methods of correlation dimension [5], RP methods makes it possible to display states of systems considered in multidimensional phase spaces in the equivalent simpler and more clearly 2-dimensional phase space. It is known that it is possible to restore a mapping of a state of complex dynamic systems by one measured coordinate of a vector of states [6]. However, this path has additional uncertainty of a dimension parameter of an enclosing in the calculation of RP. Paper [7] considers forecasting of dangerous states of the polluted atmosphere of industrial cities based on the RP calculation for one of coordinates of a vector of states. The calculation of $\mathrm{RP}$ goes for the usual metric (distance) in the space of all real numbers. The paper does not consider other types of metrics and their corresponding spaces. Authors of work [8] consider a method for calculation of RP for a 5-dimensional vector of states of wind speeds in five regions of Nigeria. They consider linear space and the Euclidean metric only. They do not consider other metrics, spaces, irregularity of measurements and adaptation of a threshold in calculating of RP. Paper [9] considers parameterization of the method for RP calculation under conditions of artifacts [9]. The paper considers the Euclidean metric of a phase space only. Authors of work [10] study methods for calculation of RP of the reconstructed vector of states of the Earth's magnetosphere [10]. However, they use only normalized linear spaces with the maximum and Chebyshev metrics as the RP calculation methods. Authors of paper [11] note that recognition and classification of human motor activity based on the calculation of RP has low reliability due to peculiarities of measurement conditions and threshold uncertainty of methods. They propose not to consider a threshold in RP methods to eliminate low reliability and errors in recognition and classification, and to calculate distance matrices directly. However, application of neural networks for recognition and classification of motor activity has well-known disadvantages and limitations. Authors of work [12] consider a method for calculation of $\mathrm{RP}$ in a space with the Euclidean metric for studying of the behavior of various biosystems. They note that measurement conditions, duration of a time delay, a dimension of an enclosing and a threshold affect reliability of the RP calculation significantly. A choice of the threshold for each set of measurements should be individual, but it should not exceed $10 \%$ of the maximum diameter of a phase space.

Paper [13] considers a method for RP calculation at irregular measurements. RP calculations are limited to space with a conventional distance metric. Work [14] considers general recommendations on overcoming of threshold uncertainty of RP methods. It proposes to overcome the threshold uncertainty by fixing the threshold, depending on a specific task of the study. Authors of paper [15] state that a threshold value should be some function of a standard deviation of measurements. They do not specify a type of function. They only note that such method of determination of a threshold can be useful for any method of RP calculation. Authors of paper [16] consider a combination of a multilevel network approach and recurrence networks for analysis of dynamics of states in multidimensional phase spaces. They study a multidimensional vector of states only in a normed space with the Euclidean metric. They do not consider other types of metrics and spaces and do not discuss irregularity of measurements and reliability of calculation of dynamics of states. Work [17] considers peculiarities of RP calculation for localization of transitional processes in systems. It notes that it is possible to use spaces with different metrics in RP calculation methods. However, it does not consider an influence of metrics and thresholds reliability of the RP calculation. Authors of paper [18] consider application of the RP method for early detection of indoor fires by a concentration of carbon monoxide [18]. They use measurements in a one-dimensional phase space with ordinary and degree distance metrics. However, they do not consider multidimensional phase spaces and threshold adaptation. They do not discuss irregularity of measurements and its influence on the RP calculation. Authors of work [19] consider methods and devices for adaptation of the threshold for identification of dangerous factors of fire [19]. They note that adaptation of a threshold is a key factor for trustworthy reliable detection [20].

Thus, a basis of RP calculation methods is finite-dimensional linear normed spaces and application of various metrics. We can consider the methods for the calculation of RP in linear spaces with a uniform, Euclidean and maximum metric as the most studied ones for now. Therefore, RP for the same system, calculated in spaces with different metrics will not be the same due to different methods of calculation of distances. Methods for calculation of RP in spaces with other types of metrics are less studied. A choice of a measure and a threshold affect reliability of mapping of recurrence states in RP. There are several heuristics known for selection of a threshold at the instant [14]. However, the considered heuristics are particular cases, which are limited to well-known metrics of spaces and have significant implementation disadvantages. In this regard, a measure and a threshold of recurrence become key factors on the way to ensure reliable mapping of recurrence states of real systems in RP [1]. Therefore, an important and unresolved part of the problem is the development of methods for the quick and 
reliable mapping of recurrence states in RP under conditions of irregular measurements.

\section{The aim and objectives of the study}

The objective of the study is to develop a method for calculation of recurrence plots to provide operative and reliable mapping of recurrence states of real dynamic systems at irregular measurements.

To accomplish the aim, the following tasks have been set:

- substantiation of a method for operative calculation of recurrence plots at irregular measurements of a vector of states to provide a reliable mapping of recurrence states of real dynamic systems;

- experimental verification of operability of the proposed method for calculation of recurrence plots on the example with irregular measurements of real dynamics of states of dangerous pollutants in the urban atmosphere.

\section{Substantiation of the method for operative calculation of recurrence plots in the presence of intervals with the absence of measurements}

The base of known methods for RP calculation is real measurements of a given $m$-dimensional $Z_{i}$ vector of states of the studied complex dynamic system during a given observation interval. Usually, measurements take place at discrete $i$ instants of a given observation interval. We can generalize the known methods for RP calculation in the form of expression

$$
\begin{aligned}
& R_{i, j}^{m, \varepsilon}=\Theta\left(\varepsilon, \Delta_{i, j}\right), \quad \Delta_{i, j}=\left\|Z_{i}-Z_{j}\right\|, \\
& Z_{i} \in \Omega^{m}, \quad Z_{j} \in \Omega^{m}, \quad i, j=0,1,2, \ldots, N_{S}-1,
\end{aligned}
$$

where $\Theta\left(\varepsilon, \Delta_{i, j}\right)$ is the Heaviside function; $\varepsilon$ is the recurrence threshold, which determines a radius of $m$-dimensional sphere of $Z_{i}$ recurrence states for $i$ time instants; $\Delta_{i, j}$ is the arbitrary norm of corresponding vector of states in $m$-dimensional space at $i$ and $j$ time instants, respectively; $N_{S}$ is the number of measurements.

Following generalization (1), the calculated RP depend on the value of $\varepsilon$ recurrence threshold, measured $Z_{i}$ and $Z_{j}$ vectors of a state of system, and the used norm. Each vector of states corresponds to a certain point in $m$-dimensional space. This means that there is $N_{S}-1$ point specified on the considered observation interval in $m$-dimensional space. Different methods for determination of the norm in (1) will lead to a different number of calculated recurrence states or recurrence points for $\varepsilon$ fixed threshold. If the method for determination of the norm is given, then the choice of a too small $\varepsilon$ threshold can lead to an absence of calculated recurrent states, although they are present in the system under study in fact. On the other hand, if the threshold $\varepsilon$ is too large, then almost every vector of states will be recurrent to any other vector of states. This means that the RP calculation of RP in accordance with expression (1) is ambiguous and therefore it is not sufficiently reliable. For most applications, it is necessary to take into account the fact that results of measurement of a vector of states at a given observation interval arrive sequentially in time, they contain noise. They also may simply be absent at certain points in time as a result of peculiarities of conditions for real measurements. In addition, following (1), a base of the RP calculation is the results of all measurements of the $m$-dimensional vector of $Z_{i}$ states at $i=0,1,2, \ldots, N_{S}-1$ instants on a given observation interval. Therefore, the calculation of RP before obtaining all measurements of the $m$-dimensional vector of states is not possible. Therefore, methods (1) in the traditional formulation are not operative, since they do not make it possible to calculate $\mathrm{RP}$ sequentially in time as measurements are taken.

Therefore, the well-known methods (1) are ambiguous, insufficiently reliable and have low efficiency of RP calculation. It is possible to ensure the speed of RP calculation in the class of methods under consideration (1) by replacing of $i, j=0,1,2, \ldots, N_{S}-1$ with the condition for real measurements, for which $i=0,1,2, \ldots, N_{S}-1$ and $j \leq i$. Based on the foregoing, following (1), we can describe $T R_{i}^{m, \varepsilon}$, class of operative methods by the following expression:

$$
T R_{i}^{m, \varepsilon}=\left\{\begin{array}{lll}
R_{i, j}^{m, \varepsilon}, & \text { if } & j \leq i, \\
0, & \text { if } & j>i,
\end{array}\right.
$$

$$
i=0,1,2, \ldots, N_{S}-1 \text {. }
$$

Expression (2) is a transformation of the corresponding class of known methods (1) into the proposed class of operative methods for the RP calculation. A base of (2) is calculation methods (1), so the proposed class of operative methods for calculation of RP is also ambiguous and insufficiently reliable. The increase in reliability of the methods of $\mathrm{RP}$ calculation (1) and (2) is important due to the fact that RP are the basis of reliability for subsequent methods for quantitative assessment of recurrence of states in real systems. However, in practice, one should not consider an increase in reliability of the RP calculation unlimited. Reliability should be sufficient to display recurrence of states, which exist in real systems. Only if we achieve enough reliability, we can guarantee a reliable quantitative assessment of recurrence of states in real systems. However, there are objective difficulties associated with internal and external factors in achievement of sufficient reliability. The internal factors, which limit reliability of RP calculated by (1) and (2) methods, are ambiguity of a choice of $\varepsilon$ threshold and a norm. External factors, which limit reliability of the RP calculation, include presence of noise and irregular measurements.

Researchers set $\varepsilon$ threshold and norms by the specific value of the threshold and the method of determination of the norm traditionally independently to overcome the ambiguity of the choice $[1,21]$. Octahedral, spherical, or cubic norms induced by uniform, Euclidean, and maximal metrics in the $m$-dimensional phase space, respectively, are the most commonly metrics. $Z_{i}$ recurrent states of the system under study in the $m$-dimensional phase space, following (1), will be graphically displayed on a two-dimensional matrix (RP). The structure of the displayed recurrence states in the matrix will depend on the selected norm of a given $\varepsilon$ threshold value. Each element of such matrix, which is equal to unity (black dot), will correspond to the conditional recurrence of a vector of states of the system under study for different values of $i$ and $j$, and zero (white point) - to the absence of recurrence. The coordinate axes of the RP representation will be time axes, which correspond to discrete instants of the vector of states measurement. The mapping of recurrence states of systems will occur in a similar way on a two-dimensional matrix for the proposed class of operative methods for 
RP calculation (2). However, unit and zero elements, which correspond to presence and absence of recurrence of states in the system, will be displayed as black and white dots in the lower triangular part of the matrix only. The upper triangular part of the matrix will have zeros only. The $T R_{i}^{m, \varepsilon}$ display matrix has triangular form because of the use of only vector of states measurements, which precedes $i$ current instant, for $\mathrm{RP}$ calculation.

It is possible to overcome a negative influence on the reliability of the RP calculation caused by noise and irregular measurements by using of heuristic methods [22, 23]. In this case, it is necessary to determine $\varepsilon$ threshold depending on the dimension of the phase space or the known model of dynamics of a vector of states of the system. However, we usually do not know the dynamics of a vector of states of real systems a priori. It can change during the observation process arbitrarily. The known methods $[22,23]$ have significant limitations under such conditions. They are not constructive to ensure sufficient reliability of the $\mathrm{RP}$ calculation, and they relate to particular cases. Irregularity of measurements in the observation interval, if we use (1) and (2), in the case of the given $\varepsilon$ threshold and the selected norm for the RP calculation will look as corresponding clusters of black dots of square shape (in case (1)) and triangular shape (in case (2)). The presence of the mentioned clusters on RP will lead to false estimates in the quantitative assessment of the recurrence of states of the studied system. Therefore, an increase in the reliability of the RP calculation is impossible without elimination of the influence of irregular measurements.

The choice of the threshold and the method of determination of the norm should be consistent to ensure sufficient reliability of the RP calculation in accordance with (1) and (2). The threshold should be adaptive to measurements of the vector of states on the observation interval. It is difficult to fulfill the indicated requirements within the framework of the known spaces and norms [1] for the RP calculation [21]. Therefore, it is necessary to improve the known spaces to introduce constructive norms, which will make possible to reconcile the norms with the threshold, and to adopt the threshold to the current measurements of a vector of states $[10,24]$. It is possible to improve spaces by introduction of an additional geometric characteristic into them - a scalar product for vector of states [24]. The scalar product of vector of states is a mapping of ordered pairs of linear space vectors onto the real axis. Let us denote such mapping for two arbitrary vectors of states of the system with $Z_{i}, Z_{j}=Z_{i}{ }^{\mathrm{T}}, Z_{j}$, where $T$ is the transposition sign. In the improved phase space, the scalar product will generate the norm for an arbitrary $Z_{i}$ vector of states in the form

$$
\left\|Z_{i}\right\|=\left(Z_{i}, Z_{j}\right)^{0,5}=\left(Z_{i}^{T}, Z_{j}\right)^{0,5} .
$$

Following (3), the following ratio determines the norm of the difference of $Z_{i}-Z_{j}$ arbitrary vector of states [24]

$$
\left\|Z_{i}-Z_{j}\right\|=\sqrt{\left(Z_{i}-Z_{j}\right)^{\mathrm{T}}\left(Z_{i}-Z_{j}\right)} .
$$

It follows from ratio (4) that the Euclidean metric, which determines the geometric distance between two arbitrary points, $Z_{i}$ and $Z_{j}$ vectors, induces the norm. If we consider (4), we can describe the proposed operative method for the RP calculation in the class of methods (2) for $m$-dimensional vectors of $Z_{i}$ states of the system under study at the current time in the space of the improved structure by the expression

$$
T R_{i}^{m, \varepsilon}=\left\{\begin{array}{lll}
\Theta\left(\varepsilon, \sqrt{\left(Z_{i}-Z_{j}\right)^{\mathrm{T}}\left(Z_{i}-Z_{j}\right)}\right), & \text { if } & j \leq i, \\
0, & \text { if } & j>i
\end{array}\right.
$$

$$
i=0,1,2, \ldots, N_{S}-1 \text {. }
$$

It follows from expression (5) that the norm of the difference of vector of states in the improved space is equivalent to the spherical norm for the Euclidean metric of the original space. An important feature of (5) is calculation of the square root of the scalar product of the difference of the corresponding vector of states of the system instead of the spherical norm. In the operative method (5), one can choose an arbitrary $\varepsilon$ threshold or determine it based on the well-known recommendations [22,23]. In contrast to the spherical norm induced by the Euclidean metric, $\sqrt{\left(Z_{i}-Z_{j}\right)^{\mathrm{T}}\left(Z_{i}-Z_{j}\right)}$ norm in (5) contains information not only about a distance between the points, which correspond to $Z_{i}$ and $Z_{j}$ vector of states of the system, but also additional information about lengths of the mentioned vectors and a relative angular position of the vectors. It is possible due to the proposed improvement in the source space. The information contained in $\left(Z_{i}-Z_{j}\right)^{\mathrm{T}}\left(Z_{i}-Z_{j}\right)$ scalar product in expression (5) makes it possible to perform adaptation of the $\varepsilon$ threshold $\varepsilon$, which is impossible in the source phase space. The essence of adaptation consists in choosing of the $\varepsilon$ threshold in accordance with the lengths of the measured $Z_{i}$ and $Z_{j}$ vector of states with a given value of the $\gamma$ angle between the vectors. Following [21, 24], we can determine the adaptive threshold by the expression

$$
\varepsilon\left(Z_{i}, Z_{j}, \gamma\right)=\sqrt{Z_{i}^{\mathrm{T}} Z_{i}+Z_{j}^{\mathrm{T}} Z_{j}-2 \sqrt{Z_{i}^{\mathrm{T}} Z} \sqrt{Z_{j}^{\mathrm{T}} Z_{j}} \cos \gamma} .
$$

Based on expression (6), the $\varepsilon\left(Z_{i}, Z_{j}, \gamma\right)$ threshold depends on the length of $Z_{i}$ and $Z_{j}$, vector of states and a given $\gamma$ value, which characterizes a mutual angular position of the vectors. The value of $\gamma$ determines a boundary mutual angular position of the vector of states, at which the vectors are still considered conditionally recurrent. Let the value of the mutual angular position of the vectors $\gamma=\pi \alpha / 180$, where $\alpha$ is the angle, which sets the acceptable angular distance between vectors of $Z_{j}$ states in degrees, at which these vectors are considered as recurrent vectors of $Z_{i}$ states at $i$ time instant. Therefore, the calculation (5) at the threshold of (6) will ensure adaptation of the RP calculation to the lengths of the measured vector of states. The proposed operative method for the RP calculation (5) will display recurrence states in the case of conical neighborhoods equivalent to the angular distance between vectors of states of the systems under study. The neighborhoods of recurrence in the form of cones make possible to coordinate the threshold with the norm in the improved phase space and to ensure adaptation of the threshold to the length of the measured vector of states. Thus, we can increase the overall reliability of the operative calculation of RP. However, adaptation of the threshold only to the length of the measured vector of states does not ensure sufficient reliability of the RP calculation fully in the case of irregular measurements, because the operative method (5) with an adaptive threshold (6) displays false recurrent states 
in the system due to missing measurements. It is possible to modify method (5) with an adaptive threshold to eliminate the indicated false recurrence states from the calculated RP. One can represent a modification of the operative method for the RP calculation with sufficient reliability at irregular measurements by expression

$$
T_{i}^{m}= \begin{cases}\Theta\left(\varepsilon\left(Z_{i}, Z_{j}, \gamma_{1}\right), \sqrt{\left(Z_{i}-Z_{j}\right)^{\mathrm{T}}\left(Z_{i}-Z_{j}\right)}\right)- & \\ -\Theta\left(\varepsilon\left(Z_{i}, Z_{j}, \gamma_{2}\right), \sqrt{\left(Z_{i}-Z_{j}\right)^{\mathrm{T}}\left(Z_{i}-Z_{j}\right)}\right), & \text { if } j \leq i, \\ 0, & \text { if } j>i,\end{cases}
$$

where $i=0,1,2, \ldots, N_{S}-1$. There is a difference of two RP calculated at adaptive thresholds, which correspond to different angular recurrence cones, used in the expression (7) for the operative calculation of RP with sufficient reliability in the case of irregular measurements. Angular cones of recurrence determined by boundary mutual angular positions of the vectors of $\gamma_{1}$ and $\gamma_{2}$ states should be enclosed. It means that $\gamma_{1}>\gamma_{2}$ condition is necessary for enclosing of cones. In this case, the value of $\gamma_{2}$ should be set from the condition for identification of recurrence states caused only by absence of measurements, and $\gamma_{1}-$ from the condition of ensuring of sufficient reliability of the RP calculation.

Thus, the proposed method (7) will provide sufficient reliability of the operative RP calculation for arbitrary measurements of the vector of states of systems taking into account noise and possible absence of measurements.

\section{Experimental verification of the method for operative calculation of recurrence plots at irregular measurements}

We tested the operability of the method for operative RP calculation on experimental data obtained during irregular measurements of exceeding of the maximum permissible concentration (MPC) of real gas pollution of the urban atmosphere. When we chose types of gas atmospheric pollutants, it was taken into account that current dangerous sources of urban atmosphere pollution are motor vehicles, fires [25, 26], and accidents at critical infrastructure facilities [27]. In addition, we also took into account the existing relationship of global atmospheric pollution with the greenhouse effect, acid rain [28], and pollution of aquifers [29]. In this regard, we chose the excess of MPC by formaldehyde $\left(\mathrm{CH}_{2} \mathrm{O}\right)$, ammonia $\left(\mathrm{NH}_{3}\right)$, and carbon monoxide $(\mathrm{CO})$ as measured components of the vector of states of the polluted atmosphere. Paper [30] presents the experimental measurement methodology and characteristics of the equipment used. The test interval for irregular measurements of the excess of MPC by atmospheric pollutants was from 13:00 on May 3, $2018(i=490)$ to 01:00 on May 11, $2018(i=520)$. We calculated a number of the $i$-th measurement of the excess of MPC of atmospheric pollutants from 13:00 on January $01,2018(i=0)$. The indicated test interval was chosen due to the presence of significant excesses of the MPC of atmospheric pollutants on it [21].

Fig. 1 illustrates RP calculated based on the classes of methods (1) and (2) with Euclidean metric and a fixed threshold $\varepsilon=2$.

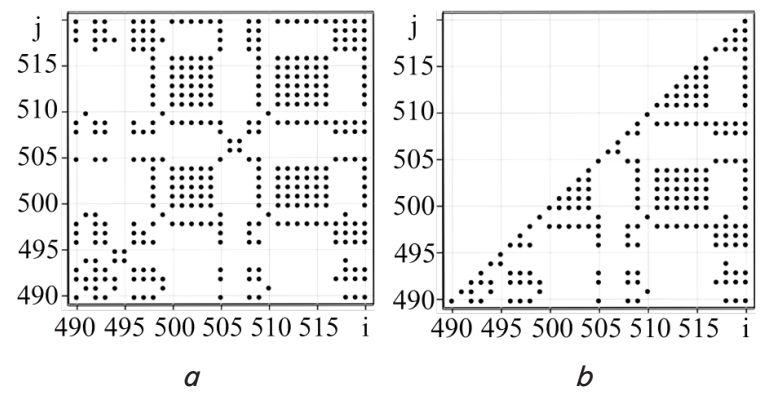

Fig. 1. RP for the vector of states of the polluted atmosphere calculated with Euclidean metric of space and a fixed threshold $\varepsilon=2$ based on the methods: $a-$ known class of methods (1); $b$ - operative class of methods (2)

Fig. 2 shows the results of application of the proposed method of the RP calculation (5) for the considered vectors of states of the polluted atmosphere on the test interval in the space of the improved structure for various values of the fixed $\varepsilon$ threshold equal to 2 and 1.

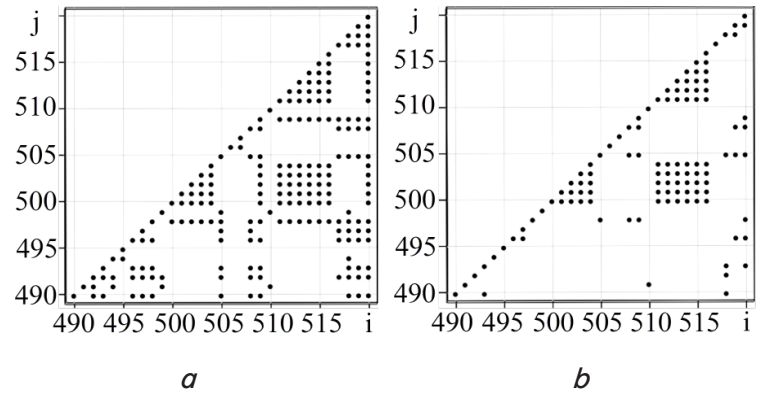

Fig. 2. RP for the vector of states of the polluted atmosphere calculated based on the operative method (5) in the space of the improved structure for various values of the fixed threshold: $a-\varepsilon=2 ; b-\varepsilon=1$

Fig. 3, $a$ shows the influence of replacement of a fixed threshold with an adaptive threshold defined by (6) at $\alpha=10^{\circ}$ in the operative method for the RP calculation (5). The red oval marks the region of atmosphere recurrence states, which characterize laminarity of the processes causing appearance of dangerous excesses of MPC. Fig. 3, $b$ illustrates the dynamics of the adaptive threshold (6) at $\alpha=10^{\circ}$.

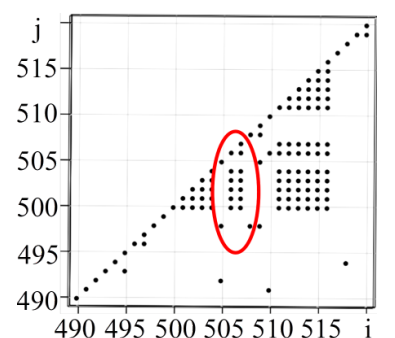

a

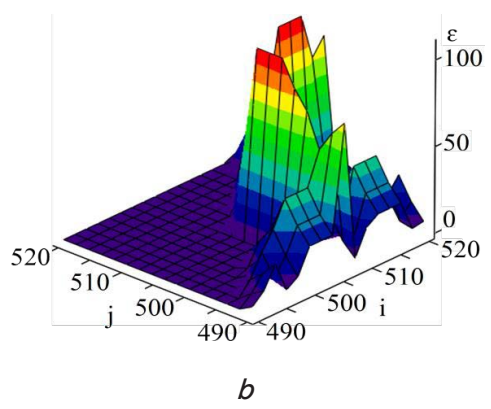

$b$
Fig. 3. RP for the vector of states of the polluted atmosphere calculated based on the operative method (5) at the adaptive threshold (6) and its dynamics: $a-\mathrm{RP} ; b-$ threshold dynamics

One can see that the value of the recurrence threshold turns out to be different and varies depending on the state of the polluted atmosphere at each measurement. 
The greatest value of the adaptive threshold corresponds to time instants when states change little (the level of red color of the surface in Fig. 3, b). Such states are characteristic to stationary conditions when there is no dispersion of pollution and its accumulation in the atmosphere. A nature of the dynamics of the adaptive threshold in time depends on the specified angle of recurrence of vectors of states. Fig. $4, b$ shows the dynamics of the adaptive threshold (6) at a larger value of $\alpha=60^{\circ}$. The dynamics of the adaptive threshold turn out to be more regular in the region of the current recurrence of states in this case. However, the adaptive threshold does not make it possible to recognize and filter false recurrence states in RP caused by a lack of measurements. Fig. 4, $a$ shows the obtained RP in the case of angles $\alpha_{1}=10^{\circ}$ and $\alpha_{2}=0.001^{\circ}$ as an illustration of reliable operative calculation of RP based on (7).

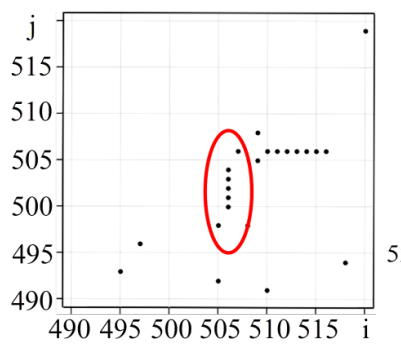

a

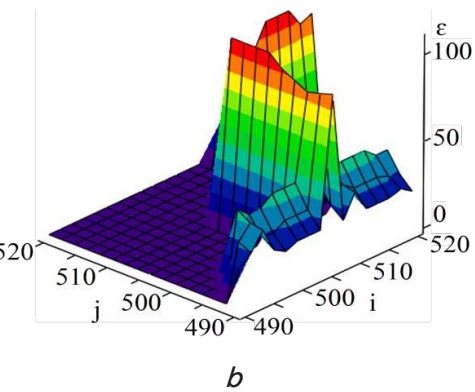

$b$
Fig. 4. Dynamics of the adaptive threshold (6) at $\alpha=60^{\circ}$ and RP for the vector of states of the polluted atmosphere (7) in the case of the adaptive threshold at $\alpha_{1}=10^{\circ}$ and $\alpha_{2}=0.001^{\circ}$ : $a-\mathrm{RP} ; b-$ threshold dynamics

The red oval in Fig. 4, $a$ shows the region of reliable recurrence states, which correspond to dangerous excesses of MPC of atmospheric gas pollution in the considered interval between 504 and 511 counting. Separate black currents in Fig. 4, $a$ indicate the presence of random recurrence states, which occur at various discrete time instants. Separate recurrence points do not indicate occurrence of dangerous states in the polluted atmosphere.

\section{Discussion of results from experimental verification of the proposed method}

We calculated RPs shown in Fig. 1 based on the methods of classes (1) and (2). Euclidean metric of spaces and a fixed threshold are characteristic to them. A comparison of RPs shows that the methods of class (2) are operative. Visually RPs in Fig. 1, $a, b$ are the same taking into account symmetry, since they are calculated for the same metric with the same fixed value of the recurrence threshold. The main difference between the methods of the RP calculation is that we need measurements over the entire specified observation interval to calculate RP shown in Fig. 1, $a$. In contrast to the class of known methods (1), a base of the operative methods (2) is the results of current and previous measurements only. Therefore, they make it possible to calculate RP in real time. If we have a given fixed threshold $\varepsilon=2$, we can see that the known and operative methods do not make it possible to reliably detect dangerous excesses in the maximum permissible concentration in the observation interval between 504 and 511 counts. In this case, one can see the measurement ir- regularity in the form of clusters of recurrence states (black points) on RP, which are false for the vector of states of the observed polluted atmosphere.

Clusters of recurrence states (black points) of a rectangular and square shape on RP are false, because their cause is not recurrence states of the studied system of atmospheric pollution, but absence of measurements (observations) and peculiarities of their representation at the indicated time instants. In this case, we cannot consider the calculated RPs as reliable and reliable mappings of recurrence states of the observed system. That is why the class of operative methods under consideration (2) requires a corresponding improvement in the direction of increasing of the required reliability and reliability of RP by elimination of the indicated false recurrence states in the $\mathrm{RP}$ calculation.

Therefore, we propose improving the initial phase space by introduction of the scalar product for vectors of states measured at discrete time instants into it. Fig. 2 illustrates RPs calculated for various values of the recurrence threshold in accordance with the operative method (5). A comparison of the obtained RPs evidences that a decrease in the threshold leads to a decrease in the number of individual recurrent states per RP. At the same time, despite the efficiency of the method, there is no mapping of dangerous excesses of the maximum permissible concentration in the interval between 504 and 511 counting on RP, and there is no elimination of false clusters of recurrence states associated with absence of measurements.

The RPs calculated in accordance with method (5) at an adaptive recurrence threshold for $\alpha=10^{\circ}$ and shown in Fig. 3, $a$ indicate advantages of using of the adaptive threshold. Such a threshold (6) makes it possible to increase reliability of the RP calculation. It makes it possible to display recurrent states of the polluted atmosphere (red oval), which correspond to dangerous atmospheric states. However, the adaptive threshold does not make it possible to eliminate false recurrence states caused by a lack of measurements. The illustration of the dynamics of the adaptive threshold in Fig. $3, b$ at $\alpha=10^{\circ}$ and in Fig. $4, b$ at $\alpha=60^{\circ}$ indicate its significant difference from the case of using of a fixed threshold in (5). One can observe a significant change in the dynamics of the adaptive threshold in the interval of dangerous excesses of MPC, which correspond to recurrence states. In this case, an increase in the boundary value of $\alpha$ angle between the vectors of states of the polluted atmosphere leads to a smoothing of the character of the dynamics, but it remains significant in the interval of dangerous excess of MPC. The diagrams in Fig. 4, $a$ indicate that the proposed method (7) provides operative reliable calculation of recurrence states of the polluted atmosphere in the absence of calculation of false recurrence clusters caused by irregular measurements. Sufficient reliability of the RP calculation makes it possible to adequately map actual dangerous states of the polluted atmosphere. The sufficient reliability of the RP calculation means the calculation of RPs, which maps adequately recurrence states caused by properties of the systems under study only, and not false states caused by measurement peculiarities. It was established that the proposed method (7) provided a reliable mapping of recurrence states of atmospheric pollution at angles $\alpha_{1}=5-10^{\circ}$ and $\alpha_{2}=$ $=0,001-5^{\circ}$ in the case of using of irregular experimental data. RPs map recurrence states inherent only to the systems under study under the described conditions. 
One should note that the well-known one-parameter spherical neighborhoods of recurrence of states transform into equivalent three-parameter conical neighborhoods of recurrence in the improved space. The new paradigm of a three-parameter conical neighborhood for recurrent states makes it possible to move on to generalized recurrence of states. The concept of generalized recurrence makes it possible to substantiate introduction of an adaptive threshold consistent with the chosen metric. The adaptive threshold provides an increase in reliability of the RP calculation. The proposed adaptive threshold ensures invariance of the RP calculation to the measured lengths of vectors of states of the system, and the angular size of a state recurrence cone provides a given accuracy for calculation of recurrence states.

We propose a method for the RP calculation based on the difference of two RPs to expand advantages of application of an adaptive threshold at irregular measurements. We calculate each of the two indicated RPs at an adaptive threshold with different accuracy. It means that it has been proposed to use two with different angular sizes of a cone instead of one conical neighborhood of recurrence. The base of the RP calculation subtraction of RP with a smaller angular cone size from RP with a larger angular cone size.

The limitations of the study include numerical results on the admissible values of the angular sizes for two cones used in the proposed method for the RP calculation, because we obtained the results for specific atmospheric pollution conditions. They are not general recommendations. However, verification of the above recommendations under other conditions and various systems suggests the general nature of the results. Therefore, confirmation or clarification of the obtained recommendations can be useful for other systems and conditions. One can consider them as a possible direction for the development of the study.

\section{Conclusions}

1. A method has been developed for the operative RP calculation at irregular measurements of a vector of states of real dynamic systems. A new scientific result was the theoretical substantiation of the method of operative RP calculation. The base of the method was improvement of the phase space for vectors of states by introduction of the scalar product operation of vectors into it. Further, we represented the space metric as a scalar product of a difference of the corresponding vectors of states. That has made it possible to coordinate a choice of the norm with the threshold, and adaption of the threshold to measurement results in order to ensure reliable mapping of recurrence states of real systems in RP. The use of two adaptive thresholds with different angular parameters made possible to develop a method for the operative calculation of RP in the case of irregular measurements. The method provided sufficient reliability of the mapping of recurrence states in RPs for applications.

2. The efficiency of the proposed operative method for calculation of recurrence plots has been demonstrated on the example with irregular measurements of the real dynamics of the vector of states of the excess of MPC of urban atmosphere pollution. We took formaldehyde, ammonia and carbon monoxide as dangerous atmospheric gas pollutants. Overall, the obtained results of the RP calculation confirmed the efficiency of the proposed method. It was established experimentally that the proposed method of the vector of states of the excess of MPC of atmospheric pollution at for irregular measurements provided a reliable mapping of recurrence states with the angular parameters of the recurrence cones $\alpha_{1}=5-10$ and $\alpha_{2}=0.001-5^{\circ}$ for the first and second adaptive thresholds, respectively.

\section{References}

1. Webber, C. L., Marwan, N. (Eds.) (2015). Recurrence Quantification Analysis. Understanding Complex Systems. Springer. doi: https://doi.org/10.1007/978-3-319-07155-8

2. Marwan, N., Webber, C. L., Macau, E. E. N., Viana, R. L. (2018). Introduction to focus issue: Recurrence quantification analysis for understanding complex systems. Chaos: An Interdisciplinary Journal of Nonlinear Science, 28 (8), 085601. doi: https://doi.org/ $10.1063 / 1.5050929$

3. Oya, S., Aihara, K., Hirata, Y. (2014). Forecasting abrupt changes in foreign exchange markets: method using dynamical network marker. New Journal of Physics, 16 (11), 115015. doi: https://doi.org/10.1088/1367-2630/16/11/115015

4. Marwan, N. (2011). How to avoid potential pitfalls in recurrence plot based data analysis. International Journal of Bifurcation and Chaos, 21 (04), 1003-1017. doi: https://doi.org/10.1142/s0218127411029008

5. Pospelov, B., Andronov, V., Rybka, E., Meleshchenko, R., Gornostal, S. (2018). Analysis of correlation dimensionality of the state of a gas medium at early ignition of materials. Eastern-European Journal of Enterprise Technologies, 5 (10 (95)), 25-30. doi: https:// doi.org/10.15587/1729-4061.2018.142995

6. Takens, F. (1981). Detecting strange attractors in turbulence. Lecture Notes in Mathematics, 366-381. doi: https://doi.org/ $10.1007 / \mathrm{bfb} 0091924$

7. Pospelov, B., Andronov, V., Rybka, E., Popov, V., Semkiv, O. (2018). Development of the method of frequencytemporal representation of fluctuations of gaseous medium parameters at fire. Eastern-European Journal of Enterprise Technologies, 2 (10 (92)), 44-49. doi: https://doi.org/10.15587/1729-4061.2018.125926

8. Adeniji, A. E., Olusola, O. I., Njah, A. N. (2018). Comparative study of chaotic features in hourly wind speed using recurrence quantification analysis. AIP Advances, 8 (2), 025102. doi: https://doi.org/10.1063/1.4998674

9. Wendi, D., Marwan, N., Merz, B. (2018). In Search of Determinism-Sensitive Region to Avoid Artefacts in Recurrence Plots. International Journal of Bifurcation and Chaos, 28 (01), 1850007. doi: https://doi.org/10.1142/s0218127418500074

10. Donner, R. V., Balasis, G., Stolbova, V., Georgiou, M., Wiedermann, M., Kurths, J. (2019). Recurrence-Based Quantification of Dynamical Complexity in the Earth's Magnetosphere at Geospace Storm Timescales. Journal of Geophysical Research: Space Physics, 124 (1), 90-108. doi: https://doi.org/10.1029/2018ja025318 
11. Garcia-Ceja, E., Uddin, M. Z., Torresen, J. (2018). Classification of Recurrence Plots' Distance Matrices with a Convolutional Neural Network for Activity Recognition. Procedia Computer Science, 130, 157-163. doi: https://doi.org/10.1016/j.procs.2018.04.025

12. Neves, F. M., Viana, R. L., Pie, M. R. (2017). Recurrence analysis of ant activity patterns. PLOS ONE, 12 (10), e0185968. doi: https://doi.org/10.1371/journal.pone.0185968

13. Ozken, I., Eroglu, D., Breitenbach, S. F. M., Marwan, N., Tan, L., Tirnakli, U., Kurths, J. (2018). Recurrence plot analysis of irregularly sampled data. Physical Review E, 98 (5). doi: https://doi.org/10.1103/physreve.98.052215

14. Souza, E. G., Viana, R. L., Lopes, S. R. (2008). Using recurrences to characterize the hyperchaos-chaos transition. Physical Review E, 78 (6). doi: https://doi.org/10.1103/physreve.78.066206

15. Schinkel, S., Dimigen, O., Marwan, N. (2008). Selection of recurrence threshold for signal detection. The European Physical Journal Special Topics, 164 (1), 45-53. doi: https://doi.org/10.1140/epjst/e2008-00833-5

16. Eroglu, D., Marwan, N., Stebich, M., Kurths, J. (2018). Multiplex recurrence networks. Physical Review E, 97 (1). doi: https://doi.org/ 10.1103/physreve.97.012312

17. Webber, C. L., Ioana, C., Marwan, N. (Eds.) (2016). Recurrence Plots and Their Quantifications: Expanding Horizons. Springer Proceedings in Physics. doi: https://doi.org/10.1007/978-3-319-29922-8

18. Pospelov, B., Andronov, V., Rybka, E., Meleshchenko, R., Borodych, P. (2018). Studying the recurrent diagrams of carbon monoxide concentration at early ignitions in premises. Eastern-European Journal of Enterprise Technologies, 3 (9 (93)), 34-40. doi: https://doi.org/10.15587/1729-4061.2018.133127

19. Pospelov, B., Andronov, V., Rybka, E., Skliarov, S. (2017). Design of fire detectors capable of self-adjusting by ignition. EasternEuropean Journal of Enterprise Technologies, 4 (9 (88)), 53-59. doi: https://doi.org/10.15587/1729-4061.2017.108448

20. Pospelov, B., Andronov, V., Rybka, E., Skliarov, S. (2017). Research into dynamics of setting the threshold and a probability of ignition detection by selfadjusting fire detectors. Eastern-European Journal of Enterprise Technologies, 5 (9 (89)), 43-48. doi: https:// doi.org/10.15587/1729-4061.2017.110092

21. Pospelov, B., Rybka, E., Togobytska, V., Meleshchenko, R., Danchenko, Y., Butenko, T. et. al. (2019). Construction of the method for semi-adaptive threshold scaling transformation when computing recurrent plots. Eastern-European Journal of Enterprise Technologies, 4 (10 (100)), 22-29. doi: https://doi.org/10.15587/1729-4061.2019.176579

22. Mindlin, G. M., Gilmore, R. (1992). Topological analysis and synthesis of chaotic time series. Physica D: Nonlinear Phenomena, 58 (1-4), 229-242. doi: https://doi.org/10.1016/0167-2789(92)90111-y

23. Thiel, M., Romano, M. C., Kurths, J., Meucci, R., Allaria, E., Arecchi, F. T. (2002). Influence of observational noise on the recurrence quantification analysis. Physica D: Nonlinear Phenomena, 171 (3), 138-152. doi: https://doi.org/10.1016/s0167-2789(02)00586-9

24. Pospelov, B., Andronov, V., Meleshchenko, R., Danchenko, Y., Artemenko, I., Romaniak, M. et. al. (2019). Construction of methods for computing recurrence plots in space with a scalar product. Eastern-European Journal of Enterprise Technologies, 3 (4 (99)), 37-44. doi: https://doi.org/10.15587/1729-4061.2019.169887

25. Vasiliev, M. I., Movchan, I. O., Koval, O. M. (2014). Diminishing of ecological risk via optimization of fire-extinguishing system projects in timber-yards. Naukovyi Visnyk Natsionalnoho Hirnychoho Universytetu, 5, 106-113.

26. Dubinin, D., Korytchenko, K., Lisnyak, A., Hrytsyna, I., Trigub, V. (2017). Numerical simulation of the creation of a fire fighting barrier using an explosion of a combustible charge. Eastern-European Journal of Enterprise Technologies, 6 (10 (90)), 11-16. doi: https://doi.org/10.15587/1729-4061.2017.114504

27. Semko, A., Rusanova, O., Kazak, O., Beskrovnaya, M., Vinogradov, S., Gricina, I. (2015). The use of pulsed high-speed liquid jet for putting out gas blow-out. The International Journal of Multiphysics, 9 (1), 9-20. doi: https://doi.org/10.1260/1750-9548.9.1.9

28. Kustov, M. V., Kalugin, V. D., Tutunik, V. V., Tarakhno, E. V. (2019). Physicochemical principles of the technology of modified pyrotechnic compositions to reduce the chemical pollution of the atmosphere. Voprosy khimii i khimicheskoi tekhnologii, 1, 92-99. doi: https://doi.org/10.32434/0321-4095-2019-122-1-92-99

29. Vasyukov, A., Loboichenko, V., Bushtec, S. (2016). Identification of bottled natural waters by using direct conductometry. Ecology, Environment and Conservation, 22 (3), 1171-1176.

30. Pospelov, B., Rybka, E., Meleshchenko, R., Borodych, P., Gornostal, S. (2019). Development of the method for rapid detection of hazardous atmospheric pollution of cities with the help of recurrence measures. Eastern-European Journal of Enterprise Technologies, 1 (10 (97)), 29-35. doi: https://doi.org/10.15587/1729-4061.2019.155027 